\title{
An adaptive algorithm to prevent SQL injection
}

\author{
Ashish John, Ajay Agarwal, Manish Bhardwaj \\ Department of Computer science and Engineering, SRM University, NCR Campus, Modinagar, Ghaziabad, India.
}

Email address:

ashishjohn@live.com (A. John), ajay.aagar@gmail.com (A. Agarwal), aapkaapna13@gmail.com (M. Bhardwaj)

\section{To cite this article:}

Ashish John, Ajay Agarwal, Manish Bhardwaj. An Adaptive Algorithm to Prevent SQL Injection. American Journal of Networks and Communications. Special Issue: Ad Hoc Networks. Vol. 4, No. 3-1, 2015, pp. 12-15. doi: 10.11648/j.ajnc.s.2015040301.13

\begin{abstract}
SQL Injection attacks are one of the top most threats for application written for the web. SQL Injection is a type of attack in which the attacker uses SQL commands to gain access or make changes to data. It allows attacker to obtain unauthorized access to the database to change the intended queries. In the web environment, end user privacy is one of the most controversial legal issues. Using SQL Injection, an attacker can leak confidential information such as credit card no. ATM Pin, User Credentials etc from the web applications or even corrupts the database. An unauthorized access to this much of confidential data by an attacker can threat to user confidentiality. In this paper, we had surveyed existing techniques against SQL Injection and analyzed their advantages and disadvantages and proposed a novel and effective solution to avoid attacks on login phase.
\end{abstract}

Keywords: SQLIA, Parse Tree Validation, Code Conversion, Static Query

\section{Introduction}

The Internet has just entered the middle Ages. The simple security model of the Stone Age still works for single hosts and LANs. But it no longer works for WANs in general and Internet in particular [1]. The lack of adequate knowledge and understanding of software and security engineering leads to security vulnerabilities, e.g. by inappropriate programming, getting even worse under deadline pressure and rush to market issues. Some solution may be effective today, but as technology changes, new risks and challenges appear. Moreover, different solutions must be combined to be effective against different types of attacks and the security of the system must be constantly monitored. A database-driven Web application commonly has four tiers namely presentation tier, logic tier, application server and data tier.

The presentation tier is the topmost level of the application. It displays information related to such services as browsing merchandise, purchasing, and shopping cart contents, and it communicates with other tiers by outputting results to the browser/client tier and all other tiers in the network.

The logic tier is pulled out from the presentation tier, and as its own layer, it controls an application's functionality by performing detailed processing.

An application server in an $n$-tier architecture is a server that hosts an application programming interface (API) to expose business logic and business processes for use by applications.

The data tier consists of database servers. Here, information is stored and retrieved. This tier keeps data independent from application servers or business logic. Giving data its own tier also improves scalability and performance.

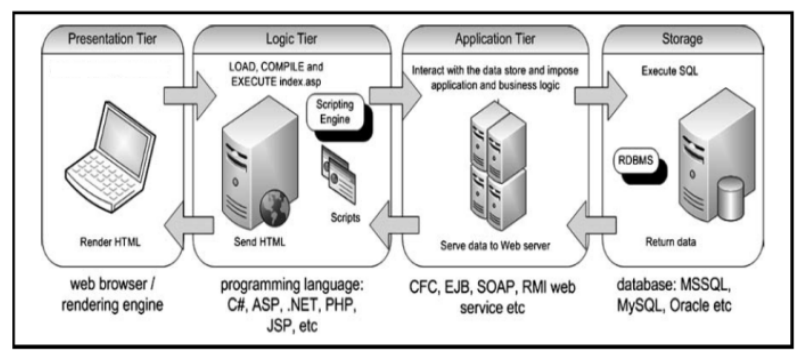

Figure 1. Database driven web application

The back-end database often contains confidential and sensitive information such security numbers, credit card number, financial data, medical data. Typically the web user supplies information, such as a username and password and web applications receive user request and interact with the back-end database and returned relevant data to the user[2]. Some of the commonly performed web attacks are: Injection attacks, XSS Attack, CSRF Attack, Security Misconfiguration etc. According to OWASP ( Open Web Application Security Project) Injection attack is at the first 
place of the top 10 web attacks that are executed in 2013[3]. SQL injection is a method for exploiting web applications that use client-supplied data in SQL queries. SQL Injection refers to the technique of inserting SQL meta-characters and commands into Web-based input fields in order to manipulate the execution of the back-end SQL queries[4]. The SQLIA occurs when an intruder changes the structure of the query by inserting any SQL commands. This paper proposes a very simple and effective method to detect SQL Injection Attacks which uses the combination of Parse Tree Validation Technique and Code Conversion Method. The rest of the paper is organized in the form of different sections. Section 2 describes the SQLIA and its categories. Section 3 discusses the related work. Section 4 explains the proposed method to detect and prevent SQLIAs. Section 5 describes the results with some discussion. Section 6 concludes this paper.

\section{SQL Injection}

SQL injection is a technique (like other web attack mechanisms) to attack data driven applications. The attacker takes the advantage of poorly filtered or not correctly escaped characters embedded in SQL statements into parsing variable data from user input. The attacker inject arbitrary data, most often a database query, into a string that's eventually executed by the database through a web application (e.g. a login form).

\subsection{SQL Injection Method}

Here are some methods through which SQL statements are injected into vulnerable systems

- Injected through user input.

- Injection through cookie fields contain attack strings..

- Injection through Server Variables.

- Second-Order Injection where hidden statements to be executed at another time by another function.

\subsection{Recent SQL Injection Attacks in NEWS}

\subsubsection{Russian Hackers Amass Over a Billion Internet Passwords \\ By NICOLE PERLROTH and DAVID GELLES - AUG. 5, 2014}

A Russian crime ring has amassed the largest known collection of stolen Internet credentials, including 1.2 billion user name and password combinations and more than 500 million email addresses, security researchers say.

The records, discovered by Hold Security, a firm in Milwaukee, include confidential material gathered from 420,000 websites, including household names, and small Internet sites. Hold Security has a history of uncovering significant hacks, including the theft last year of tens of millions of records from Adobe Systems.

\subsection{2. “NASDAQ is owned." Five Men Charged in Largest Financial Hack Ever \\ By Dan Goodin - July 262013}

Five Eastern European men have been charged with operating a global hacking operation that infiltrated some of the world's biggest financial institutions, pilfered data for more than 160 million credit cards, and created hundreds of millions of dollars in losses.

\subsubsection{SQL Injection Used in the Largest Data Security Breach in U.S. History to Date}

Posted on August 20, 2009 by acunetix

Three men, responsible for the largest data security breach in U.S. history, stole 130 million credit and debit card numbers from five leading companies. They took advantage of a coding error, and allegedly used a SQL injection attack to compromise a web application, which was used as the starting point to help them bypass company network firewalls and gain access over companies' networks.

\subsection{SQLIA Types}

There are various types of SQL Injection available. Some them that are highly user are described and explained here with there SQL codes and explanation.

\subsubsection{Tautologies Attack}

Purpose :

- Identify injectable parameters

- Bypass authentication

- Extract data

In logic, a tautology is a formula which is true in every possible interpretation. In a tautology-based attack the code is injected using the conditional OR operator such that the query always evaluates to TRUE. Tautology-based SQL injection attacks are usually bypass user authentication and extract data by inserting a tautology in the WHERE clause of a SQL query. The query transform the original condition into a tautology, causes all the rows in the database table are open to an unauthorized user. A typical SQL tautology has the form "or <comparison expression>", where the comparison expression uses one or more relational operators to compare operands and generate an always true condition.

Eg.:

Attacker's Input:

User ID: abcd

Password: anything' or ' 1 '='1

Backend Process:

Select * from table where userid='abcd' and pass='anything' or ' 1 '=' 1 ';

\subsubsection{Piggy-Backed Queries / Statement Injection Attack}

Purpose :

- Extract data

- Modify dataset

- Execute remote commands

- Denial of service

This type of attack is different than others because the hacker inject additional queries to the original query, as a result the database receives multiple SQL queries. The first query is valid and executed normally, the subsequent queries are the injected queries, which are executed in addition to the 
first. Due to misconfiguration a system is vulnerable to piggy-backed queries and allows multiple statements in one query.

Attacker's Input:

User ID: abcd

Password: ' ; drop table details --

Backend Process:

Select * from table where userid='abcd' and pass="'; drop table details -- ;

\subsubsection{Union Query}

Purpose :

- Bypassing authentication

- Extract data

This type of attack can be done by inserting a UNION query into a vulnerable parameter which returns a dataset that is the union of the result of the original first query and the results of the injected query. The SQL UNION operator combines the results of two or more queries and makes a result set which includes fetched rows from the participating queries in the UNION.

Basic rules for combining two or more queries using UNION :

1) Number of columns and order of columns of all queries must be same.

2) The data types of the columns on involving table in each query should be same or compatible.

3) Usually returned column names are taken from the first query.

By default the UNION behalves like UNION [DISTINCT], i.e. eliminated the duplicate rows; however, using ALL keyword with UNION returns all rows, including duplicates. The attacker who try to use this method must have solid knowledge of DB schema.

Attacker's Input:

User ID: ' union select * from details--

Password: abcd

Backend Process:

Select $*$ from table where userid=" union select $*$ from details -- and pass='abcd';

\subsubsection{Illegal/Logically Incorrect Queries}

Purpose :

- Identify injectable parameters

- Identify database

- Extract data

In this type of injection an attacker is try gather information about the type and structure of the back-end database of a Web application. The attack is considered as preliminary step for further attacks. If an incorrect query is sent to a database, some application servers returns the default error message and the attacker takes the advantage of this weakness. They inject code in vulnerable or injectable parameters which creates syntax, type conversion, or logical error. Through type error one can identify the data types of certain columns. Logical error often expose the names of tables and columns.

Attacker's Input: date: $29 \mathrm{a} / 10 / 2014$

Generated Error:

PLS-00306: wrong number or types of arguments in call to 'DETAILS'

ORA-06550: line 1, column 7:

* from this error attacker receives table name (details) and database (oracle) being used.

\subsubsection{Stored Procedures}

Purpose :

- Privilege escalation

- Denial of service

- Execute remote commands

A stored procedure is a subroutine available to applications that access a relational database system. Extensive or complex processing that requires execution of several SQL statements is moved into stored procedures, and all applications call the procedures. One can use nested stored procedures by executing one stored procedure from within another. Stored procedures type of SQL injection try to execute store procedures present in the database. Most of the database have standard set of procedures (apart from user defined procedures) that extend the functionality of the database and allow for interaction with the operating system. The attacker initially try to find the database type with other injection method like illegal/logically incorrect queries. Once an attacker determine which databases is used in backend then he try to execute various procedures through injected code. As the stored procedure are written by developers, therefore these procedures does not make the database vulnerable to SQL injection attacks. Stored procedures can be vulnerable to execute remote commands, privilege escalation, buffer overflows, and even provide administrative access to the operating system. If an attacker injects ';SHUTDOWN; -into either the User ID or Password fields then it will generate the following SQL code :

Attacker's Input:

User ID: abcd

Password: ' ;SHUTDOWN;--

Backend Process:

Select * from table where userid='abcd' and pass="'; SHUTDOWN;--

\section{Related Work}

\subsection{Parse Tree Validation Technique [5]}

The technique is based on comparing, at run time, the parse tree of the SQL statement before inclusion of user input with that resulting after inclusion of input.

\subsection{Code Conversion Method [6]}

1. Converting User input to code like ASCII, binary, hexa etc.

2. Searching the availability of converted input in Data table and returns valid Userid and Password. 


\section{Proposed Method}

The proposed method consists of the best features of both parse tree validation technique and code conversion method. In this method we parse the user input and check whether its vulnerable, if there is any chance of vulnerability present then code conversion will be applied over that input. In this way, we can detect and prevent SQL Injection using a single code. Below is the algorithm for the proposed method:

For web pages that saves data to the database:

- Take the input text.

- Apply Parse Tree Validation Technique.

- Check if Vulnerable(ie if tree size mismatch)

1. If vulnerable

2. Apply code conversion (say, ascii to binary )

3. Counter $=1$

4. If not vulnerable

5. Counter $=0$

- Save to database

- Exit

For web pages that only retrieves from the database:

- Check the value of counter

1. If counter $=0$

2. If counter $=1$

$>$ Apply reverse code conversion(say, binary to ascii)

- Display the text

- Exit

From the value of counter we can come to know whether the user input is converted or not.

\section{Result and Discussion}

After the implementation of various types of SQL injection Attacks the results received showed how important and crucial data is received by modifying the query. This loss of data causes loose to a company in millions. We had implemented various attacks in order to get an in depth knowledge of how these attacks work. Then the results are obtained after implementing the attacks. After studying and implementing some of the available methods we had come to the below mentioned result:

- Code Conversion to each and every user input is more time consuming as well as the database size will also increase.

- Parse tree validation technique will raise false alarm even if legitimate user is having blank space in his/her input.

Since available methods are not sufficient on their own to stop SQL injection attacks so in the present scenario more than one method is used so as to ensure higher security level.

\section{Conclusion and Future Work}

By conducting a comprehensive survey on existing techniques, we have realized that many SQL injection countermeasures have their limitations. Understanding and identifying the working mechanisms, as well as advantages and disadvantages of current techniques will benefit the work in this area. We will try to combine the available SQL injection prevention methods to get a higher level of security.

Besides the text field, SQL Injection attacks can be performed through cookies or through server variables. As this work is a part of my M. Tech. thesis work, our future work will be preventing SQLI attacks that are being performed by any other mean.

\section{References}

[1] Oppliger, R., "Internet security enters the Middle Ages," Computer , vol.28, no.10, pp.100,101, Oct 1995 doi: $10.1109 / 2.467613$

[2] http://www.w3resource.com/sql/sql-injection/sql-injection.php

[3] www.owasp.org

[4] W.G.J. Halfond, A. Orso, "AMNESIA: analysis and monitoring for Neutralizing SQL-injection attacks," $20^{\text {th }}$ IEEE/ACM International Conference on Automated Software Engineering, Long Beach, CA, USA, 2005, pp. 174-183.

[5] Michele Spagnuolo,Politecnico di Milano,Milan "Using Parse Tree Validation to Prevent SQL Injection Attacks"

[6] Indrani Balasundaram, E. Ramaraj "An Efficient Technique for Detection and Prevention of SQL Injection Attack using ASCII Based String Matching" International Conference on Communication Technology and System Design 2011 (C) 2011 Published by Elsevier Ltd. Selection and/or peer-review under responsibility of ICCTSD 2011

[7] Shruti Bangre, Alka Jaiswal "SQL Injection Detection and Prevention Using Input Filter Technique" International Journal of Recent Technology and Engineering (IJRTE) ISSN: 22773878, Volume-1, Issue-2, June 2012

[8] Jaskanwal Minhas and Raman Kumar "Blocking of SQL Injection Attacks by Comparing Static and Dynamic Queries" I. J. Computer Network and Information Security, 2013, 2, 1-9 Published Online February 2013 in MECS (http://www.mecspress.org/) DOI: 10.5815/ijcnis.2013.02.01

[9] W. Halfond, J. Viegas, and A. Orso. A Classification of SQLInjection Attacks and Countermeasures. Proceedings of the IEEE International Symposium on Secure Software Engineering (ISSSE), 2006.

[10] "A Survey of SQL Injection Defense Mechanisms By Kasra Amirtahmasebi", Seyed Reza Jalalinia and Saghar Khadem, Chalmers University of Technology, Sweden Presented at: Institute of Electrical and Electronics Engineers in 2009

[11] William G.J. Halfond, Jeremy Viegas, and Alessandro Orso "A Classification of SQL Injection Attacks and Countermeasures". 